\title{
Rapid and visual detection of Lawsonia intracellularis with an improved recombinase polymerase amplification assay combined with a lateral flow dipstick
}

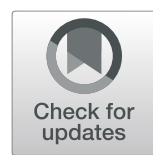

Yanyang Wu, Kaiyue Tian, Yuhan Zhang, Huifang Guo, Ning Li, Zeng Wang ${ }^{*}$ and Jun Zhao*(D)

\begin{abstract}
Background: Lawsonia intracellularis (L. intracellularis) is the etiologic agent of porcine proliferative enteropathy (PPE), which is reported in many swine breeding countries all over the world, and has caused enormous economic losses in intensive pig production systems. Therefore, the aim of this study was to develop a simple and rapid method for on-site detection of Lawsonia intracellularis (L. intracellularis). As the isothermal recombinase polymerase amplification (RPA) can be performed at a constant temperature and its product is directly observed on a lateralflow dipstick (LFD) with naked eyes without electrophoresis, the RPA-LFD assay should be useful for field diagnosis of $L$. intracellularis as well as its detection from clinical samples.

Results: The established RPA-LFD assay could be finished in $30 \mathrm{~min}$ at a wide temperature range of 25 to $40^{\circ} \mathrm{C}$, and the amplicons could be visualized by naked eyes. The developed RPA-LFD assay was specific to dnaA gene of L. intracellularis, and did not detect nucleic acids extracted from other common gastrointestinal pathogens. The minimum detection of this RPA-LFD method was $400 \mathrm{~L}$. intracellularis per reaction, which was as sensitive as conventional PCR. Further, the RPA-LFD assay was performed with 150 clinical fecal samples and the detection results were compared with conventional PCR. Results showed that the coincidence rate of RPA-LFD and conventional PCR was $100 \%$.
\end{abstract}

Conclusions: The combined RPA with LFD assay provides a simple, rapid, specific and sensitive alternative for field diagnosis of $L$. intracellularis infection.

Keywords: Lawsonia intracellularis, Recombinase polymerase amplification, Lateral flow dipstick, On-site detection

\section{Background}

Lawsonia intracellularis, a fastidious and obligate intracellular bacterium, belongs to the bacterial genus Lawsonia and is the etiologic agent of porcine proliferative enteropathy (PPE), which is characterized clinically by mild to severe diarrhea, retarded growth and/or sudden death in fattening pigs and macroscopically by thickening of the intestinal mucosa due to enterocyte proliferation [1, 2]. Since the first case of $L$. intracellularis infection was reported in 1931 [3], the prevalence of PPE has been reported in many swine breeding countries

\footnotetext{
*Correspondence: wzzxz20140105@126.com; zhaoj@henau.edu.cn College of Animal Science and Veterinary Medicine, Henan Agricultural University, Zhengzhou, Henan, China
}

all over the world, and has caused enormous economic losses in intensive pig production systems [4-9]. In China, $L$. intracellularis was first isolated from the intestinal mucosa of infected pigs in Southern China in 2008 [10]. The overall seroprevalence of $L$. intracellularis in Chinese pig herds was $77 \%$, evaluated by a blocking ELISA in 2014. A higher seroprevalence was found in fattening pigs, sows and boars compared with pre-weaning piglets and weaners [11]. To control the infection and spread of the L. intracellularis effectively, simple, rapid, sensitive and accurate methods suitable for field detection of $L$. intracellularis are critical.

The diagnosis of L. intracellularis is based on the demonstration of the microbe or microbial DNA in tissue 
specimens or fecal samples, or the demonstration of $L$. intracellularis-specific antibodies in sera. Current available diagnostic methods for L. intracellularis infection in live animals include serological tests for detecting antibody against $L$. intracellularis, such as enzyme-linked immunosorbent assay (ELISA) [12-14], immunoperoxidase monolayer assay (IPMA) [15], indirect immunofluorescence assay (IFA) [16], and methods for detecting L. intracellularis in fecal samples, such as polymerase chain reaction (PCR)-based tests [17-24] and IFA [25]. Among these methods, bacteria maintenance in vitro has limited the use of serological tests, since the cultivation of the obligate intracellular $L$. intracellularis requires establishment of suitable cell lines and L. intracellularis cannot be cultured in conventional cell-free medium. Although PCR-based molecular diagnostic tools are sensitive and capable of detecting $L$. intracellularis from various clinical samples, these techniques are restricted to the laboratory due to the requirements for sophisticated equipment, complex experimental procedures, skilled personnel and subsequent analysis. PPE may also be diagnosed postmortem from the typical macroscopic lesions, but histological confirmation by immunohistochemistry (IHC) test is needed [26-28]. Since L. intracellularis infection does not show specific clinical signs of illness at the earlier stage of infection, which often leads to the missing diagnosis. Hence there is an urgent need for a rapid and reliable pen side diagnostic assay for a better detection and control of this economically important disease of swine.

Recombinase polymerase amplification (RPA) is a novel isothermal amplification technology which couples isothermal recombinase-driven primer targeting of template material with strand-displacement DNA synthesis [29]. It overcomes the technical difficulties posed by current DNA amplification methods. RPA does not require a stringent incubation temperature for optimal performance and can achieve exponential amplification without pretreatment of sample DNA. The RPA reactions are sensitive, specific, rapid and applicable at constant low temperature ranging from 25 to $43^{\circ} \mathrm{C}$ [30]. Lateral flow dipstick (LFD) is a portable diagnostic tool which can be used for detecting various kinds of substances in non-laboratory situations. The workflow does not require highly qualified personnel and the results can be inspected by the naked eyes. LFD has been successfully incorporated into various nucleic acids based methods [31-35]. This unique combination of properties is a significant advance in the development of portable and widely accessible nucleic acid-based tests.

In this study, RPA coupled with LFD system (RPA-LFD) was developed to detect $L$. intracellularis DNA in fecal samples for the first time. The specificity and sensitivity of the RPA-LFD were evaluated. And the effectiveness of the RPA-LFD was determined by detecting clinical fecal samples.

\section{Results}

Usability of the primers

The specificity of the RPA primers was determined by BLAST search (http://www.ncbi.nlm.nih.gov/blast/Blast. cgi). As expected, the primer combination was $100 \%$ identical to sequences of dnaA gene of $L$. intracellularis from pigs (GenBank accession nos. CP004029 and AM180252). The RPA basic reaction was performed by using unlabeled primers at $37^{\circ} \mathrm{C}$ for $25 \mathrm{~min}$ to test the usability of the primers. The result of amplification showed that the 292-bp target gene could be successfully amplified. The sequencing results of RPA products were in good agreement with the dnaA gene of $L$. intracellularis. However, the target gene could not be amplified from DNA sample negative for L. intracellularis and a non-template control (Fig. 1A).

\section{Optimal reaction conditions for RPA-LFD assay}

For the adaptation to the LFD detection system, a labeling RPA was carried out. Instead of labeling lateral flow probe, a pair of labelled forward and reverse primers was used. The sequences of labelled primers were identical to those of the RPA basic primers except for containing FITC and Biotin at the $5^{\prime}$ end of the forward and reverse primer, respectively.

Based on the RPA basic reaction results, the optimal conditions of combining the RPA with lateral flow detection were determined with a consideration on the Test line signal strength, sensitivity and no Test line for $L$. intracellularis negative sample. The optimal concentration of labelled primers was determined first. The results of LFD detection showed that amplification with $1.0 \mu \mathrm{M}$ of each labelled forward and reverse primer yielded the strongest Test line for $L$. intracellularis positive sample and no Test line for L. intracellularis negative sample (Fig. 1B).

By using the optimized primer concentration, the RPA temperature and reaction time were determined. Results of the amplification showed that, within the temperature range of $30-42{ }^{\circ} \mathrm{C}$, the $L$. intracellularis DNA could be successfully amplified, and the optimal temperature range for the RPA was $37-42^{\circ} \mathrm{C}$ (Fig. 1C). The routine temperature of $37^{\circ} \mathrm{C}$ was chosen as the optimal RPA-LFD temperature.

Regarding the RPA reaction time, the results of LFD detection showed that the amplification signals appeared at $5 \mathrm{~min}$ after the start of the reaction. Amplifications for 5-30 min all yielded single and stable Test bands, and $15 \mathrm{~min}$ was chosen as the optimal RPA-LFD reaction time (Fig. 1D).

\section{Specificity of RPA-LFD assay}

In order to evaluate the specificity of the L. intracellularis-specific RPA-LFD, the RPA was carried out by using $10 \mathrm{ng}$ nucleic acids from each of the most common 


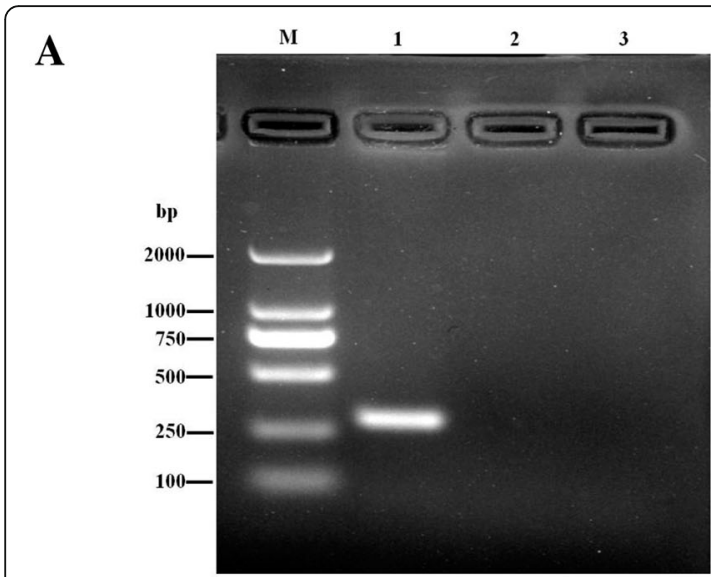

Amplification of dnaA gene fragment of $L$. intracellularis by recombinase polymerase amplification assay

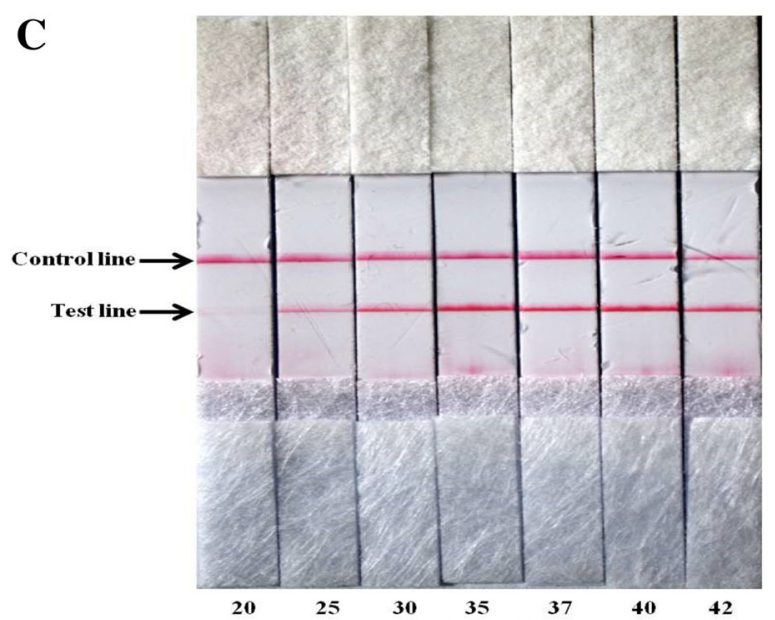

Reaction temperature for RPA-LFD $\left({ }^{\circ} \mathrm{C}\right)$

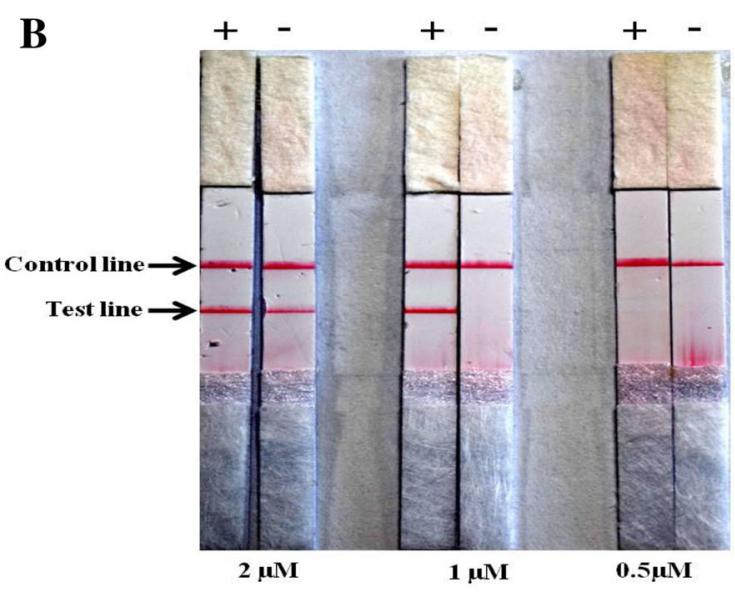

Concentration of primers for RPA-LFD

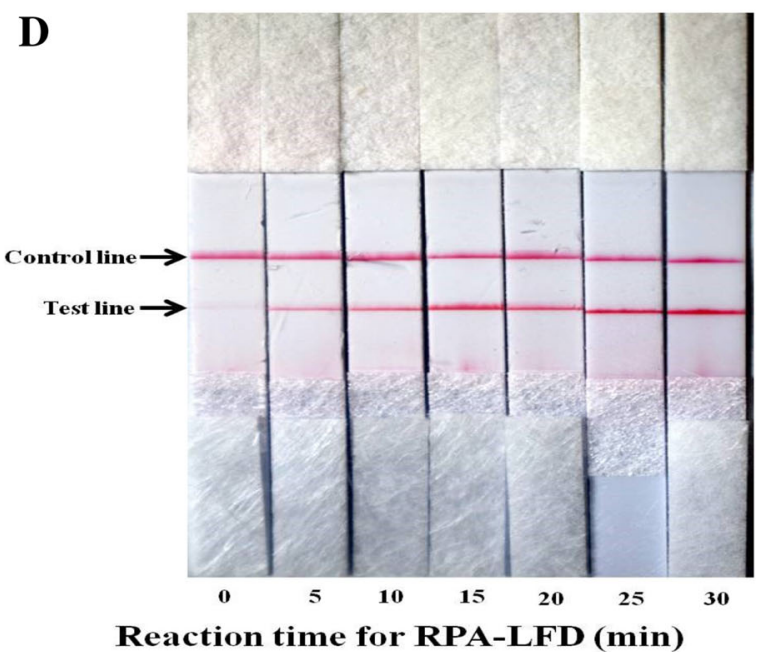

Reaction time for RPA-LFD (min)

Fig. 1 Specific amplification of dnaA gene and optimization of condition for $L$. intracellularis RPA-LFD. The dnaA gene fragment of $L$. intracellularis was specifically amplified by recombinase polymerase amplification (RPA) using designed primers (a); Concentration of primers (b), reaction temperature (c), and reaction time (d) were optimized for RPA, and the amplicons were detected using lateral flow dipstick (LFD). M: DL2000 DNA Marker; 1: L. intracellularis genomic DNA; 2: E. coli genomic DNA; 3: Blank control. L. intracellularis DNA positive samples (+) and negative samples (-) were used as templates in RPA, respectively

porcine gastrointestinal pathogens, including $E$. coli, $S$. Cholerasuis, E. faecalis, B. hyodysenteriae, PCV2, PRV, PoRV, PEDV, TGEV, CSFV, samples containing $10 \mathrm{ng}$ nucleic acid of each above pathogens except for nucleic acid of L. intracellularis as well as a mixture of $10 \mathrm{ng}$ nucleic acid of L. intracellularis and $10 \mathrm{ng}$ nucleic acid of each above pathogens as templates, and the amplicons were detected by the LFD. As shown in Fig. 2A, except for DNA extracted from a mixture of L. intracellularis and the above pathogens, only red color Control line was observed on the LFD strips. The results showed that the RPA-LFD could discriminate L.intracellularis from other common porcine gastrointestinal pathogens. The existence of L.intracellularis and other common porcine gastrointestinal pathogens in the mixed sample was demonstrated by PCR using primer sets in Table 1 (Fig. 2B).

\section{Sensitivity of RPA-LFD assay}

The analytical sensitivity of the RPA-LFD was determined by using genomic DNA extracted from pure-cultured and number-determined L. intracellularis and feces sample spiked with pure-cultured and number-determined $L$. intracellularis. As shown in Fig. 3, the established RPA-LFD had a same detection threshold of $400 \mathrm{~L}$. intracellularis per reaction for both pure-cultured L. intracellularis and feces sample spiked with pure-cultured $L$. intracellularis. When the sensitivity of the RPA-LFD was compared with that of conventional PCR, the RPA-LFD was found as sensitive as the conventional PCR. 


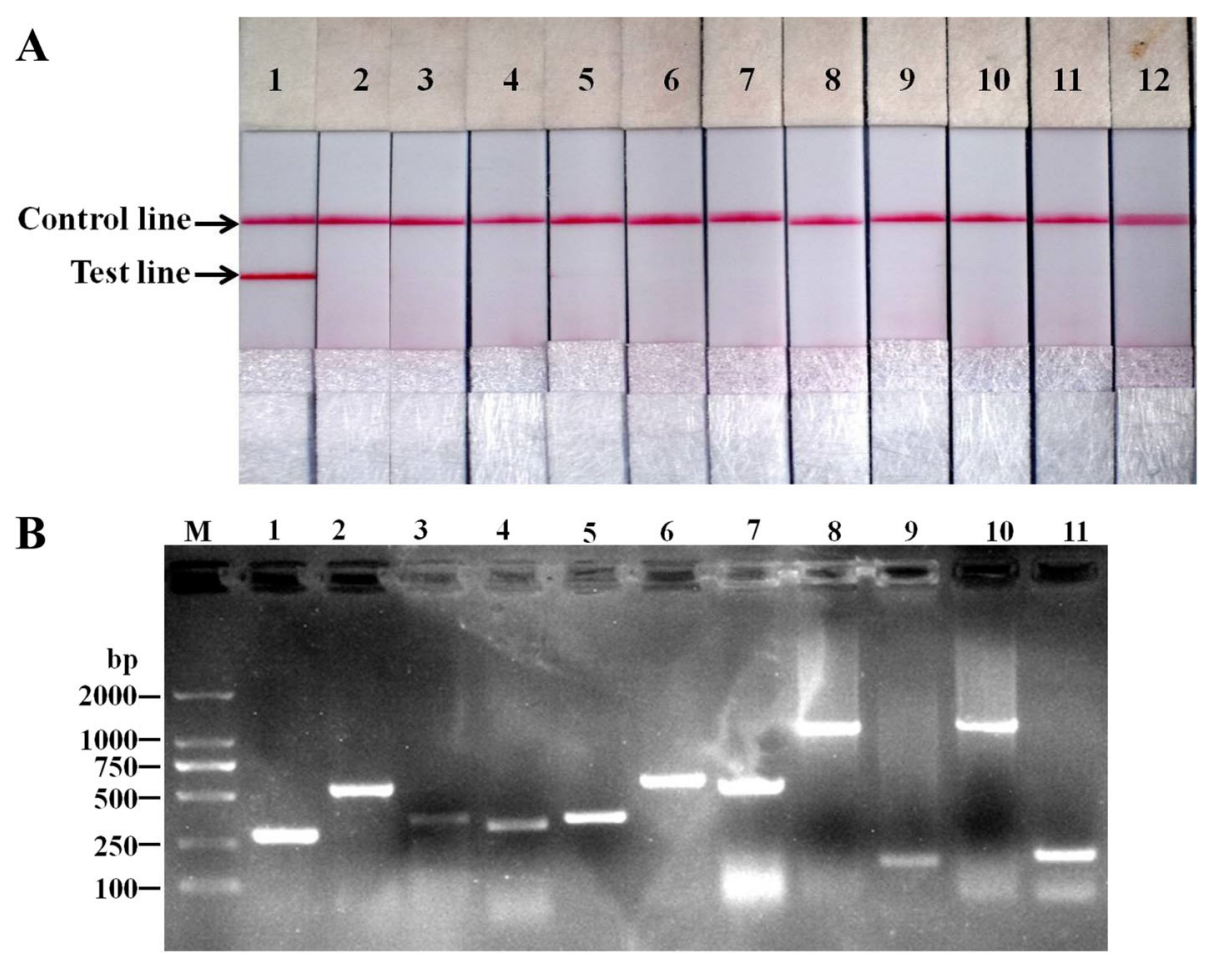

Fig. 2 Specificity of the RPA-LFD assay. a The analytical specificity test demonstrated that DNAs from E. coli, Salmonella Cholerasuis (S. Cholerasuis), Enterococcus faecalis (E. faecalis), Brachyspira hyodysenteriae (B. hyodysenteriae), porcine circovirus serotype 2 (PCV2), pseudorabies virus (PRV) and porcine rotavirus (PORV), and RNAs from porcine epidemic diarrhea virus (PEDV), porcine transmissible gastroenteritis virus (TGEV), classical swine fever virus (CSFV), and sample containing the above pathogens expect for $L$. intracellularis showed no cross-reactions in the developed L. intracellularis RPA-LFD (Lane 2-12), only sample containing both L. intracellularis and the above pathogens produced positive Test line (Lane 1); b Specific amplification L. intracellularis, E. coli, S. Cholerasuis, E. faecalis, B. hyodysenteriae, PCV2, PRV, PoRV, PEDV, TGEV, and CSFV by PCR using nucleic acids extracted from sample containing the above pathogens (Lane 1-12)

\section{Clinical performance of $L$. intracellularis RPA-LFD}

The clinical performance of the $L$. intracellularis-specific RPA-LFD was evaluated by using 150 clinical fecal samples from growing pigs suffering from diarrhea and poor performance. At the same time, the samples were tested by using conventional PCR. For each experiment, a $L$. intracellularis positive (fecal sample containing pure-cultured $L$. intracellularis from a commercial vaccine) and a negative control (fecal sample from $L$. intracellularis-negative healthy pigs detected by PCR) were included to ensure the test would not report a false positive. As shown in Fig. 4 and Table 2, the $L$. intracellularis-specific RPA-LFD could efficiently detect the $L$. intracellularis in clinical feces, and the detection results were in $100 \%$ coincidence with those of conventional PCR.

\section{Discussion}

L. intracellularis is one of the most economically important pathogens in the swine production. It causes chronic diarrhea and poor performance in young growing pigs. Simple, rapid, sensitive and accurate methods suitable for field detection of $L$. intracellularis are crucial to efficiently control the infection and the spread of the $L$. intracellularis in time. Diagnosis of L. intracellularis is usually based on the demonstration of the microbe or microbial DNA in tissue specimens or fecal samples by PCR-based methods, or the detection of $L$. intracellularis-specific antibodies in sera by serological assays. However, the current diagnostic methods have several unneglectable limitations, such as antigen availability, the need of trained personnel to conduct the diagnostics, handling of the complex equipment, shipment and storage of samples to a distant laboratory. RPA is relatively new isothermal amplification method with the support of recombinase, a strand-displacement polymerase and a single-strand binding protein. The RPA does not need special instruments and can amplify target DNA to detectable level in less time and at lower temperature than those of other DNA amplification techniques. A lateral flow dipstick (LFD) is a visual testing tool that eliminates the need for trained personnel and expensive equipments. Therefore, the platform composed by RPA and LFD shows multiple advantages, such as convenient operation, rapid reaction, easy visualization of result and less equipments and skilled personnel requirement, which make it ideal for field diagnosis of $L$. intracellularis infection. 
Table 1 Primer sequences for common porcine gastrointestinal pathogens

\begin{tabular}{|c|c|c|c|}
\hline Pathogens & primers $\left(5^{\prime}-3^{\prime}\right)$ & $\begin{array}{l}\text { product length } \\
\text { (base pair) }\end{array}$ & GenBank accession no. \\
\hline \multirow[t]{2}{*}{ L. intracellularis } & F:AAATCCAAAAGTCGAGTATCTAACTGCGG & 292 & AM180252 \\
\hline & R:TAAAAACCCAGAGCAAAATCGTGATACCAGGCG & & \\
\hline \multirow[t]{2}{*}{ E. coli } & F:AACGGTTGCTCTTCATTTAG & 678 & CP034794 \\
\hline & R:GACCATCCAATAAGTGTG & & \\
\hline \multirow[t]{2}{*}{ S. Cholerasuis } & F:GCTCTTTCGTCTGGCATTA & 351 & СР034819 \\
\hline & R:AACTTCATCGCACCGTCA & & \\
\hline \multirow[t]{2}{*}{ E. faecalis } & F:AAAGTAGAATTAGATCCACAC & 320 & CP031027 \\
\hline & R:TCTATCACATTCGGTTGCG & & \\
\hline \multirow[t]{2}{*}{ B. hyodysenteriae } & F:ACTAAAGATCCTGATGTATTTG & 352 & СР019600 \\
\hline & R:CTAATAAACGTCTGCTGC & & \\
\hline \multirow[t]{2}{*}{ PCV2 } & F:GGTGCCCGCTGCCACATCGAGAAAG & 589 & MH718995 \\
\hline & R:AGCTTCTACAGCTGGGACAGCAG & & \\
\hline \multirow[t]{2}{*}{ PRV } & F:GAGTACGTCACGGTCATCAAGGAG & 553 & NC006151 \\
\hline & R:CACTTCCGGTTTCTCCGGATC & & \\
\hline \multirow[t]{2}{*}{ PoRV } & F:GGCTTTAAAAGAGAGAATTTCCG & 1062 & JF835112 \\
\hline & R:GGTCACATCATACAATTCTAA & & \\
\hline \multirow[t]{2}{*}{ PEDV } & F:CATGGGCTAGCTITCAGGTC & 182 & MK288006 \\
\hline & R:CGGCCCATCACAGAAGTAGT & & \\
\hline \multirow[t]{2}{*}{ TGEV } & F:TCTITCTTCTACCCTATTATGACTG & 1117 & KT696544 \\
\hline & R:CTYTGGAGTATGGTGGTGTTC & & \\
\hline \multirow[t]{2}{*}{ CSFV } & F:GGTGGTCTAAGTCCTGAGTACAGG & 177 & MK121886 \\
\hline & R:GCCTCTGCAGCACCCTATCAGGTCG & & \\
\hline
\end{tabular}

L. intracellularis is an obligate intracellular bacterium that is difficult to culture, and its detection and quantification pose a challenge for epidemiological and infection studies [16]. In this study, a rapid RPA-LFD assay was developed as an alternative diagnostic method of $L$. intracellularis for the first time. Two labelled primers targeting the dnaA gene of $L$. intracellularis were designed. The RPA reaction generated a dual-tagged DNA amplicon which could be visualized on the LFD with naked eyes by untrained personnel. This is especially important for areas where instruments and trained veterinary workers are deficient. Regarding the specificity of this RPA-LFD method, there was no cross-reaction with other usual porcine gastrointestinal bacteria and viruses. The sensitivity of the RPA-LFD was evaluated and compared with conventional PCR by using pure-cultured number-determined $L$ intracellularis in this study. Since there is currently no gold standard method for the detection of L. intracellularis, and L. intracellularis cannot be cultured on artificial bacteriological media, it is hard to document accurately the specificity and sensitivity of the RPA test without samples confirmed by a gold standard test or bacteria isolation and identification. However the RPA-LFD could discriminate L.intracellularis from other common porcine gastrointestinal pathogens. Based on this result, the specificity of the RPA-LFD is $100 \%$, even only one known L.intracellularis positive sample was included. Our results showed that the established RPA-LFD had a same detection threshold of $400 \mathrm{~L}$. intracellularis per reaction for both pure-cultured $L$. intracellularis and feces sample spiked with pure-cultured L. intracellularis. Moreover, the RPA-LFD was found as sensitive as the conventional PCR. We believe that samples containing more than $400 \mathrm{~L}$. intracellularis can be $100 \%$ detected by the RPA-LFD. To mimic the field detecting condition and to test whether fecal-PCR inhibitors were affecting RPA amplification and LFD detection, we also evaluated the sensitivity of the RPA-LFD by using DNA extracted from feces sample spiked with pure-cultured numberdetermined L. intracellularis as templates for RPA-LFD and conventional PCR. The detection limit of the RPA-LFD turned out to be $400 \mathrm{~L}$. intracellularis per reaction in both cases. However, the specific bands amplified in conventional PCR from spiked feces samples were fainter than that of amplified from samples containing same number pure-cultured L. intracellularis (Fig. 3B). The above results suggest a decreased susceptivity to inhibition in RPA-FLD from feces in contrast to a greater susceptivity to inhibition 

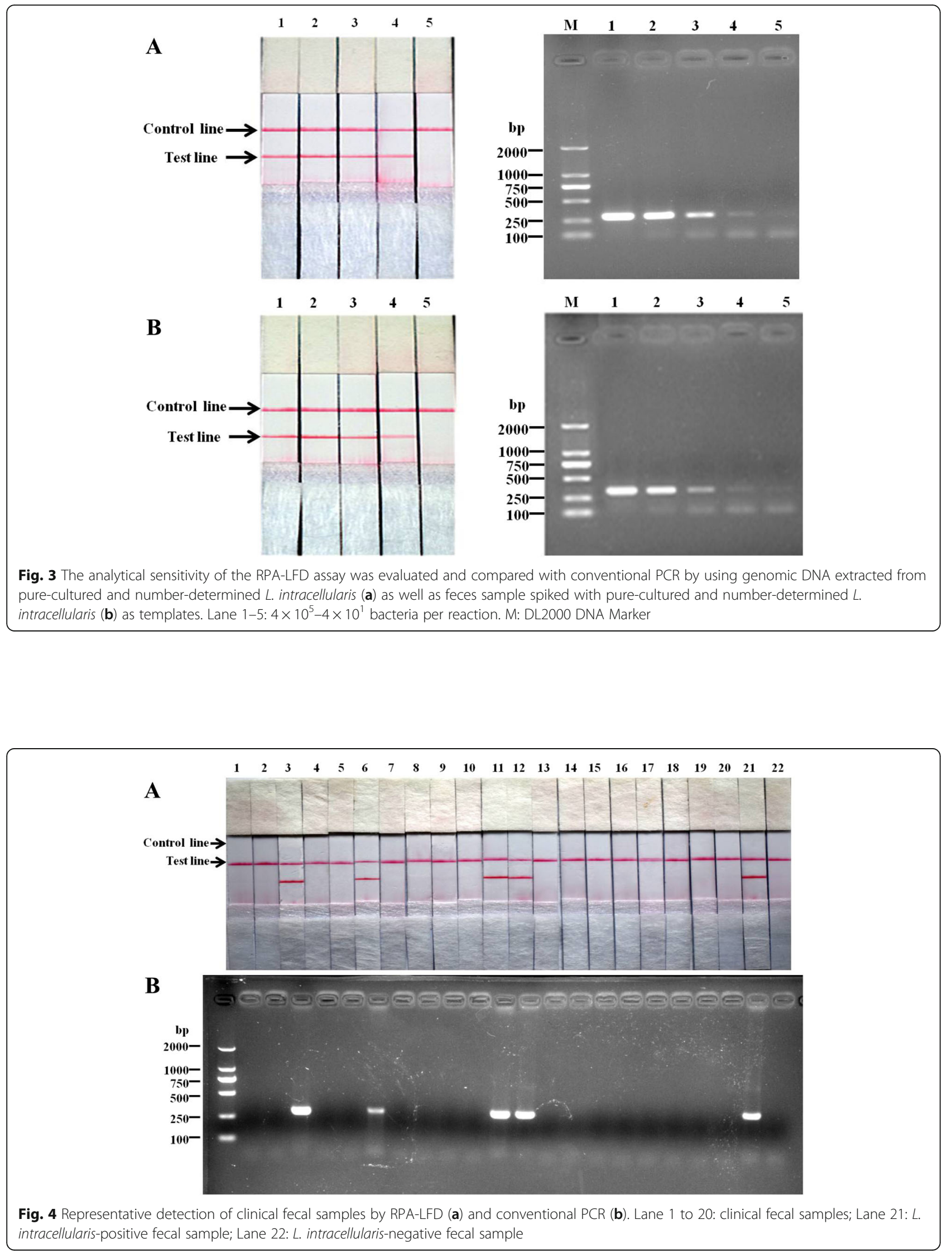
Table 2 Coincidence rate of RPA-LFD and conventional PCR

\begin{tabular}{llllll}
\hline & & \multicolumn{2}{l}{ PCR } & & CR \\
\cline { 3 - 5 } & & Positive & Negative & Total & \\
\hline RPA-LFD & Positive & 90 & 0 & 90 & \\
& Negative & 0 & 60 & 60 & $100 \%$ \\
& Total & 90 & 60 & 150 & \\
\hline
\end{tabular}

$C R$ Coincidence rate. $C R=[(90+60) / 150] \times 100 \%$

in conventional PCR. Previous study has demonstrated that disease symptoms corresponded with higher fecal shedding of $L$. intracellularis detected by a SYBR green quantitative polymerase chain reaction (qPCR) assay after inoculating $L$. intracellularis-free pigs with $5 \times 10^{5}$ bacteria per pig [20]. Thus, the sensitivity in term of number of $L$. intracellularis in feces sample presented in this study provided a means to monitor L.intracellularis shedding from naturally or experimentally infected pigs. Given the sensitivity of RPA-LFD was comparable to that of conventional PCR in detecting clinical feces samples, the RPA-LFD still had several advantages over the conventional PCR, such as more convenient operation, more rapid reaction, less equipment requirement and the results could be visualized by naked eyes.

The additional advantages of the $L$. intracellularis RPA-LFD included the elimination of the requirement of designing a special labelled probe, the shorter incubation time and lower single incubation temperature. Our study showed that the RPA-LFD assay could efficiently detect L. intracellularis from clinical fecal samples using two labelled primers rather than two primers and one probe in conventional RPA. The RPA-LFD assay could amplify target DNA at a relatively low, constant temperature from 25 to $42{ }^{\circ} \mathrm{C}$, whereas the optimum reaction temperature was 30 to $42^{\circ} \mathrm{C}$. This means that a simple heating device, such as water bath or even body heat, can be used to achieve amplification and detection. The RPA-LFD assay could amplify the target DNA to detectable levels within 5 to $10 \mathrm{~min}$, which was much shorter than those of conventional PCR-based methods. Currently, most of the reported RPA assays were developed by using commercial RPA kit from the TwistDx Company. The products of commercially available TwistAmp $^{\text {tw }}$ nfo kit can be detected by the LFD. However compatible probe is needed for the TwistAmp ${ }^{\mathrm{Tm}}$ nfo kit. A TwistAmp ${ }^{\text {Tw }}$ LF probe consist of an oligonucleotide homologous to the target sequence which incorporates a 5 ' -antigenic label, an internal abasic nucleotide analogue (a tetrahydrofuran residue or THF) which replaces a conventional base found in the target, and a polymerase extension blocking group (such as a phosphate, C3-spacer or a dideoxynucleotide) at the 3' end, and the probe is typically $46-52$ nucleotides long, at least 30 of which are placed 5' to the THF site, and at least a further
15 are located 3' to it [29]. Though the probe enhances the specificity of the RPA amplification, the probe is always hard to be found in a target gene and is expensive to be synthesized. Real-time loop-mediated isothermal amplification (RT-LAMP) has been developed for the detection of L. intracellularis [36]. However, the L. intracellularis RPA-LFD was faster than RT-LAMP (15 min in L. intracellularis RPA compared to $30-60 \mathrm{~min}$ in RT-LAMP). Moreover, RPA-LFD in this study only required a single pair of primers, while RT-LAMP needed at least 6 primers.

The diagnostic validation of the $L$. intracellularis RPA-LFD assay was evaluated with 150 clinical fecal samples and compared it with PCR. The reason we chose fecal samples is that $L$. intracellularis was always shed from feces of sick swine and the feces were easily acquired and non-invasive. As the sensitivity of RPA-LFD was comparable to that of conventional PCR in detecting clinical feces samples, the RPA-LFD results were in $100 \%$ coincidence with those of conventional PCR. Even though the conventional PCR is also suitable to detect $L$. intracellularis, the RPA-LFD still have multiple unique advantages as stated above.

In this study, the RPA templates could be prepared from clinical fecal samples with magnetic bead-based extraction methods. Compared with the traditional nucleic acid preparation methods, extraction using magnetic beads is easy and time saving, and does not require potentially dangerous procedures or specialized laboratory equipments [37]. The whole sample preparation and nucleic acid isolation process could be finished in $30 \mathrm{~min}$. The combining the RPA-LFD with magnetic bead-based nucleic acid extraction techniques makes $L$. intracellularis on-site detecting possible.

\section{Conclusion}

In summary, the established L. intracellularis RPA-LFD demonstrated multiple advantages, such as high sensitivity, good specificity, convenient operation, rapid reaction and less equipment requirement, which was suitable not only for pen side diagnosis but also for field screening and monitoring of the L. intracellularis infection. This technique can be used for epidemiological surveillance activities for $L$. intracellularis.

\section{Methods}

\section{Primer design}

Based on the published genome sequence of $L$. intracellularis PHE/MN1-00 strain (GenBank accession no. AM180252), a pair of specific primers targeting the dnaA (ATPase involved in DNA replication initiation) gene was designed for RPA: 5' - AAATCCAAAAGTCG AGTATCTAACTGCGG-3'; reverse primer: 5' - TAAA AACCCAGAGCAAAATCGTGATACCAGGCG-3'. The 
size of amplicon is $292 \mathrm{bp}$. In order to detect the RPA products by the LFD assay, the forward and reverse primers were labelled at the $5^{\prime}$-end with Fluorescein isothiocyanate (FITC) and Biotin, respectively.

\section{Nucleic acid extraction}

Genomic DNA/RNA was extracted from L. intracellularis (a commercial live attenuated $L$. intracellularis vaccine, Enterisol ${ }^{\circ}$ ileitis, Boehringer Ingelheim Vetmedica, Germany) and Escherichia coli (E. coli), Salmonella Cholerasuis (S. Cholerasuis), Enterococcus faecalis (E. faecalis), Brachyspira hyodysenteriae (B. hyodysenteriae) (ATCC-27164), porcine circovirus serotype 2 (PCV2), pseudorabies virus (PRV) Bartha-K61 strain, porcine rotavirus (PoRV), porcine epidemic diarrhea virus (PEDV) CV777 strain, transmissible gastroenteritis virus (TGEV) PUR46-MAD strain, classical swine fever virus (CSFV) vaccine $C$ strain, samples containing the above pathogens except for L. intracellularis and samples containing all the above pathogens by using the genesig ${ }^{\circ}$ DNA/RNA Extraction Kit (Primerdesign Ltd., United Kingdom) according to the manufacturer's instructions. Briefly, $200 \mu \mathrm{l}$ of sample were mixed well with $200 \mu \mathrm{l}$ of lysis buffer and $20 \mu$ l of Proteinase $K$ solution in a microcentrifuge tube. Then $500 \mu \mathrm{l}$ magnetic beads/binding buffer were added to the lysed sample, mixed well and then separated by using a magnetic separator. The separated beads were washed by using Wash Buffer 1 and 2 and $80 \%$ ethanol, respectively. Add $50 \mu \mathrm{l}$ Elution Buffer to the tube and resuspend the beads completely by repeated pipetting up and down. Then separate the magnetic beads from the sample and transfer the supernatant containing the purified DNA/RNA to a new tube and used for RPA. The protocol for extracting total DNA/RNA from clinical fecal samples is as follows: add $500 \mu \mathrm{l}$ of Sample Prep Solution to approximately 10-20 $\mathrm{mg}$ of feces (a match head in size) or $200 \mu \mathrm{l}$ if using liquid feces, and mix vigorously by shaking for $1 \mathrm{~min}$. Allow the particles to settle down. Use up to $200 \mu \mathrm{l}$ supernatant for the above extraction protocol.

\section{RPA basic reaction}

RPA basic reaction was performed to test the usability of the primers with the TwistAmp Basic kit (TwistDx, UK) according to the manufacturer's instruction. Briefly, the RPA reaction with a final reaction volume of $50 \mu \mathrm{l} \mathrm{com-}$ prised of $29.5 \mu \mathrm{l}$ rehydration buffer, $12 \mu \mathrm{ldd} \mathrm{d}_{2} \mathrm{O}, 2 \mu \mathrm{l}$ of each primer $(10 \mu \mathrm{M})$ and $2 \mu \mathrm{l}$ template. To initiate the reaction $2.5 \mu \mathrm{l}$ of magnesium acetate $(280 \mathrm{mM})$ was added. Vortex and flip the reaction tube by hand or spin the reaction tube in a palm centrifuge. Reactions were performed at $37^{\circ} \mathrm{C}$ in a water bath for $25 \mathrm{~min}$. The amplification products were purified with QIAquick PCR Purification Kit (Qiagen, Germany) and analyzed on 2\% agarose gel electrophoresis. For each experiment, a $L$. intracellularis negative (nucleic acid extracted from clinical feces which were negative for $L$. intracellularis determined by conventional PCR) and a blank control were included.

\section{RPA-LFD assay}

For lateral flow dipstick detection of the RPA products, the forward and reverse primers for RPA were labelled at the 5 '-end with FITC and Biotin, respectively. Different concentration of primers, reaction duration ranging from $5 \mathrm{~min}$ to $30 \mathrm{~min}$, and reaction temperatures ranging from $37^{\circ} \mathrm{C}$ to $42{ }^{\circ} \mathrm{C}$ were evaluated to optimize the condition for the RPA-LFD assay. After amplification, the RPA products were detected by lateral flow strip (Ustar Biotechnologies, Hangzhou, China) according to the instruction of manufacturer. The lateral flow strip contains streptavidin-coated colloidal gold on the sample pad, anti-FITC antibody on the Test line and biotin on the Control line. The result was considered valid if a pink line at the Control line was visible. The result was considered to be positive when the both Control line and pink Test line appeared.

\section{Evaluation of RPA-LFD}

Based on the optimized conditions for RPA-LFD, the sensitivity of the RPA-LFD was evaluated and compared with that of the conventional PCR by using genomic DNA extracted from pure-cultured and number-determined $\left(10^{7}, 10^{6}, 10^{5}, 10^{4}\right.$, and $10^{3}$ bacteria) L. intracellularis attenuated vaccine strain B3903 (a commercial live attenuated $L$. intracellularis vaccine, Enterisol ileitis, Boehringer Ingelheim Vetmedica, Germany). To mimic the field detecting condition, the sensitivity of the RPA-LFD was also evaluated and compared with that of the conventional PCR by using genomic DNA extracted from feces sample spiked with pure-cultured and number-determined $L$. intracellularis $\left(10^{7}, 10^{6}, 10^{5}, 10^{4}\right.$, and $10^{3}$ bacteria). The extracted DNA was dissolved in $50 \mu \mathrm{l}$ Elution buffer, and $2 \mu \mathrm{l}$ of extracted genomic DNA was used as templates for RPA-LFD and conventional PCR, respectively. Then the number of $L$. intracellularis per reaction was calculated as follow: bacterium number $\left(10^{7}-10^{3}\right) / 50 \mu \mathrm{l} \times 2 \mu \mathrm{l}$, i.e. $4 \times 10^{5}-4 \times 10^{1}$ bacteria per reaction. The PCR assay was performed in a $50 \mu \mathrm{l}$ reaction containing $25 \mu \mathrm{l}$ of $2 \times$ Es Taq MasterMix (Beijing ComWin Biotech, Beijing, China), $2 \mu \mathrm{l}$ of each primer $(10 \mu \mathrm{M}), 2 \mu \mathrm{l}$ of template, $20 \mu \mathrm{l}$ of $\mathrm{dd}_{2} \mathrm{O}$. The cycling parameters were $95^{\circ} \mathrm{C}$ for 5 min, followed by 30 cycles of denaturation at $95^{\circ} \mathrm{C}$ for 30 s, annealing at $56^{\circ} \mathrm{C}$ for $30 \mathrm{~s}$, extension at $72^{\circ} \mathrm{C}$ for 20s, and a final extension of $72^{\circ} \mathrm{C}$ for $10 \mathrm{~min}$. The PCR was performed on a Bio-Rad T100 Thermal Cycler (Bio-Rad, USA). After amplification, the PCR products 
were analyzed using agarose gel electrophoresis; and the RPA products were detected by the LFD.

Since the most common porcine gastrointestinal pathogens including E. coli, S. Cholerasuis, E. faecalis, B. hyodysenteriae, PCV2, PRV,PoRV, PEDV, TGEV, and CSFV, are frequently detected in clinical feces from pigs with diarrhea in China, the specificity of the $L$. intracellularis-specific RPA-LFD was determined with DNA or RNA samples from these gastrointestinal pathogens. The 10 ng nucleic acids from each pathogen, samples containing the above pathogens except for L. intracellularis as well as a mixture of $10 \mathrm{ng}$ nucleic acids from pure L. intracellularis and $10 \mathrm{ng}$ nucleic acids from the above each gastrointestinal pathogens were respectively tested. The existence of each pathogen was demonstrated by conventional PCR using the primers in Table 1.

Clinical fecal samples from growing pigs suffering from diarrhea and poor performance were used to evaluate the diagnostic validity. For each experiment, a $L$. intracellularis positive (fecal sample containing pure-cultured $L$. intracellularis from a commercial vaccine) and a negative control (fecal sample from $L$. intracellularis-negative healthy pigs detected by PCR) were included to ensure the test would not report a false positive.

\section{Abbreviations}

B. hyodysenteriae: Brachyspira hydysenteriae; CSFV: Classical swine fever virus; E. faecalis: Enterococcus faecalis; E.coli: Escherichia coli; ELISA: Enzyme-linked immunosorbent assay; IFA: Indirect immunofluorescence assay; IHC: Immunohistochemistry; IPMA: Immunoperoxidase monolayer assay; L. intracellularis: Lawsonia intracellularis; LFD: Lateral flow dipstick; PCR: Polymerase chain reaction; PCV2: Procine circovirus serorype2; PEDV: Porcine epidemic diarrhea virus; PoRV: Porcine rotavirus; PPE: Porcine proliferative enteropathy; PRV: Pseudorabies virus; qPCR: Quantitative polymerase chain reaction; RPA: Recombinase polymerase amplification; RTLAMP: Real-time loop-mediated isothermal amplification;

S.Cholerasuis: Salmonella Cholerasuis; TGEV: Transmissible gastroenteritis virus

\section{Acknowledgements}

The authors thank the farmers and veterinarians who helped the collection and delivery of pig fecal samples.

\section{Funding}

The study was supported by the National Key R\&D Program of China (NO 2016YFD0501100).

\section{Availability of data and materials}

The dataset analyzed during the current study is available from the corresponding author on reasonable request.

\section{Author contributions}

YYW designed and drafted the work and wrote the manuscript. YYW, KYT, YHZ, $H F G$, and NL performed the experiments, analyzed the data, and interpreted the results. ZW conceived and interpreted experiments. JZ helped conceive project and edit the manuscript. All authors approved the final version.

\section{Ethics approval and consent to participate}

This study was approved by the Animal Ethics Committee of Henan Agricultural University. Experimental protocols for obtaining swine clinical samples used in this study were carried out in strict accordance with the Animal Ethics Procedures and Guidelines of the People's Republic of China.
Consent for publication

Not applicable.

\section{Competing interests}

No conflict of interest declared.

\section{Publisher's Note}

Springer Nature remains neutral with regard to jurisdictional claims in published maps and institutional affiliations.

Received: 8 November 2018 Accepted: 10 March 2019

Published online: 21 March 2019

\section{References}

1. Lawson GH, Gebhart CJ. Proliferative enteropathy. J Comp Pathol. 2000; 122(2-3):77-100

2. McOrist S, Gebhart CJ, Boid R, Barns SM. Characterization of Lawsonia intracellularis gen. Nov., sp. nov., the obligately intracellular bacterium of porcine proliferative enteropathy. Int J Syst Bacteriol. 1995;45(4):820-5.

3. Biester HE, Schwarte LH. Intestinal adenoma in swine. Am J Pathol. 1931; 7(2):175-185 176

4. Stege H, Jensen TK, Moller K, Baekbo P, Jorsal SE. Prevalence of intestinal pathogens in Danish finishing pig herds. Preventive veterinary medicine. 2000;46(4):279-92.

5. Jacobson M, Gerth LM, Holmgren N, Lundeheim N, Fellström C. The prevalences of Brachyspira spp. and Lawsonia intracellularis in Swedish piglet producing herds and wild boar population. J Vet Med. 2005;52(9): 386-91.

6. Suh DK, Song JC. Prevalence of Lawsonia intracellularis, Brachyspira hyodysenteriae and Salmonella in swine herds. J Vet Sci. 2005;6(4):289.

7. Lee SW, Kim TJ, Park SY, Song CS, Chang HK, Yeh JK, Park HI, Lee JB. Prevalence of porcine proliferative enteropathy and its control with tylosin in Korea. J Vet Sci. 2001;2(3):209-12.

8. Holyoake PK, Emery D, Gonsalves J, Donahoo M, Collins A. Prevalence of antibodies to Lawsonia intracellularis in pig herds in Australia. Aust Vet $\mathrm{J}$. 2010;88(5):186-8.

9. Armbruster GA, Deen J, Gebhan CJ, Pelger GA, Keffaber KK, Parks CW. Review of Lawsonia intracellularis seroprevalence screening in the United States, June 2003 to July: Elanco animal health, greenfield. Indiana.

10. Xiao AH, Xie LH, Liao CQ, Lan JN, Li MN, Hou SY, Lu SY, Huang WJ. Analysis of antigenicity in four antigenic candidate genes of Lawsonia intracellularis GXNN strain. Guangxi Agricultural Sciences. 2010:62-5.

11. Wu Z, Ling Y, Tian D, Pan Q, Heegaard PMH, He C. Seroprevalence of Lawsonia intracellularis antibodies in intensive pig farms in China. BMC Vet Res. 2014:10(1):100.

12. Holyoake PK, Cutler RS, Caple IW, Monckton RP. Enzyme-linked immunosorbent assay for measuring ileal symbiont intracellularis-specific immunoglobulin G response in sera of pigs. J Clin Microbiol. 1994;32(8): 1980-5.

13. Boesen HT, Jensen TK, Møller K, Nielsen LH, Jungersen G. Evaluation of a novel enzyme-linked immunosorbent assay for serological diagnosis of porcine proliferative enteropathy. Vet Microbiol. 2005;109(1):105-12.

14. Magdalena J, Per W, Ann N, Malik M, UIf E. Evaluation of a blocking ELISA for the detection of antibodies against Lawsonia intracellularisin pig sera. Acta Vet Scand. 2011;53(1):1-6.

15. Guedes RM, Gebhart CJ, Deen J, Winkelman NL. Validation of an immunoperoxidase monolayer assay as a serologic test for porcine proliferative enteropathy. Journal of Veterinary Diagnostic Investigation Official Publication of the American Association of Veterinary Laboratory Diagnosticians Inc. 2002;14(6):528.

16. Guedes RM, Gebhart CJ, Winkelman NL, Mackienuss RA. A comparative study of an indirect fluorescent antibody test and an immunoperoxidase monolayer assay for the diagnosis of porcine proliferative enteropathy. Journal of Veterinary Diagnostic Investigation Official Publication of the American Association of Veterinary Laboratory Diagnosticians Inc. 2002; 14(5):420

17. Jones GF, Ward GE, Murtaugh MP, Lin G, Gebhart CJ. Enhanced detection of intracellular organism of swine proliferative enteritis, ileal symbiont intracellularis, in feces by polymerase chain reaction. J Clin Microbiol. 1993; 31(10):2611-5. 
18. Mcorist S, Gebhart CJ, Lawson GH. Polymerase chain reaction for diagnosis of porcine proliferative enteropathy. Vet Microbiol. 1994;41(3):205.

19. Richter B, Ladinig A, Nedorost N, Weissenböck H. A TaqMan quantitative polymerase chain reaction assay for the detection of Lawsonia intracellularis in fecal and tissue samples from pigs. J Veterinary Diagnostic Investigation Official Publication Am Association of Veterinary Laboratory Diagnosticians Inc. 2010;22(1):70.

20. Drozd M, Kassem II, Gebreyes W, Rajashekara G. A quantitative polymerase chain reaction assay for detection and quantification of Lawsonia intracellularis. J Vet Diagn Investig. 2010;22(2):265-9.

21. Jordan DM, Knittel JP, Roof MB, Schwartz K, Larson D, Hoffman L. Detection of Lawsonia intracellularis in swine using polymerase chain reaction methodology. J Veterinary Diagnostic Investigation Official Publication of the American Association of Veterinary Laboratory Diagnosticians Inc. 1999;11(1):45.

22. Nathues H, Holthaus K, Grosse Beilage E. Quantification of Lawsonia intracellularis in porcine faeces by real-time PCR. J Appl Microbiol. 2009; 107(6):2009-16.

23. Suh DK, Lym SK, Bae YC, Lee KW, Choi WP, Song JC. Detection of Lawsonia intracellularis in diagnostic specimens by one-step PCR. J Vet Sci. 2000;1 (1):33

24. Wattanaphansak S, Gebhart CJ, Anderson JM, Singer RS. Development of a polymerase chain reaction assay for quantification of Lawsonia intracellularis. J Vet Diagn Investig. 2010;22(4):598-602.

25. Just SD, Thoen CO, Thacker BJ, Thompson JU. Monitoring of Lawsonia intracellularis by indirect serum immunofluorescence assay in a commerical swine production system. J Swine Health \& Production. 2001;9(2):57-61.

26. Junghyun K, Changsun C, Wanseob C, Chanhee C. Immunohistochemistry and polymerase chain reaction for the detection of Lawsonia intracellularis in porcine intestinal tissues with proliferative enteropathy. J Vet Med Sci. 2000;62(7):771.

27. Mcorist S, Boid R, Lawson GH, Mcconnell I. Monoclonal antibodies to intracellular campylobacter-like organisms of the porcine proliferative enteropathies. Vet Rec. 1987;121(18):421-2.

28. Szczotka A, Stadejek T, Zmudzki J, Nowak A, Osiński Z, Pejsak Z. Immunohistochemical detection of Lawsonia intracellularis in tissue sections from pigs. Pol J Vet Sci. 2011;14(4):531-8.

29. Piepenburg O, Williams CH, Stemple DL, Armes NA. DNA detection using recombination proteins. PLoS Biol. 2006;4(7):1115-21.

30. Lorraine L, Dara L, Singhal MC, Jason C, Jered S, Paul L, Anthony T, Olaf P, Mathew P, Robert W. Non-instrumented incubation of a recombinase polymerase amplification assay for the rapid and sensitive detection of Proviral HIV-1 DNA. PLoS One. 2014;9(9):e108189.

31. Cordray MS, Richards-Kortum RR. A paper and plastic device for the combined isothermal amplification and lateral flow detection of Plasmodium DNA. Malar J. 2015;14(1):472.

32. Jaroenram W, Owens L. Recombinase polymerase amplification combined with a lateral flow dipstick for discriminating between infectious Penaeus stylirostris densovirus and virus-related sequences in shrimp genome. J Virol Methods. 2014;208:144-51.

33. Rigano LA, Malamud F, Orce IG, Filippone MP, Marano MR, Amaral AMD, Castagnaro AP, Vojnov AA. Rapid and sensitive detection of Candidatus Liberibacter asiaticus by loop mediated isothermal amplification combined with a lateral flow dipstick. BMC Microbiol. 2014;14(1):86.

34. Sun $K$, Xing W, Yu X, Fu W, Wang Y, Zou M, Luo Z, Xu D. Recombinase polymerase amplification combined with a lateral flow dipstick for rapid and visual detection of Schistosoma japonicum. Parasit Vectors. 2016; 9(1):476.

35. Yongkiettrakul S, Kampeera J, Chareanchim W, Rattanajak R, Pornthanakasem W, Kiatpathomchai W, Kongkasuriyachai D. Simple detection of single nucleotide polymorphism in Plasmodium falciparum by SNP-LAMP assay combined with lateral flow dipstick. Parasitol Int. 2017; 66(1):964-71.

36. Byungyong $P$, Kwanseob S, Wonil K, Hossain MM, Bumseok K, Jungkee $K$, Choikyu P, Sungjin C, Inho J, Hoseong C. Rapid and sensitive detection of Lawsonia intracellularis in pigs by real-time loop-mediated isothermal amplification. Acta Veterinaria. 2015;65(1):20-9.

37. Caldarellistefano R, Vago L, Bonetto S, Nebuloni M, Costanzi G: Use of magnetic beads for tissue DNA extraction and IS6110 mycobacterium tuberculosis PCR. Mol Pathol Mp 1999, 52(3):158.

Ready to submit your research? Choose BMC and benefit from:

- fast, convenient online submission

- thorough peer review by experienced researchers in your field

- rapid publication on acceptance

- support for research data, including large and complex data types

- gold Open Access which fosters wider collaboration and increased citations

- maximum visibility for your research: over $100 \mathrm{M}$ website views per year

At BMC, research is always in progress.

Learn more biomedcentral.com/submissions 